\title{
Erratum: Theory of reflectivity properties of graphene-coated material plates [Phys. Rev. B 92, 125419 (2015)]
}

\author{
G. L. Klimchitskaya, C. C. Korikov, and V. M. Petrov \\ (Received 6 April 2016; published 20 April 2016)
}

DOI: 10.1103/PhysRevB.93.159906

There are several technical errors in this paper that have no impact on the obtained results. On the right-hand side of Eq. (10), the multiple $i$ in front of the second line should be replaced with $-i$. As a result, Eqs. (19) and (20) take the form

$$
\begin{aligned}
\Pi_{00}\left(\omega, \theta_{i}\right) & \approx-8 \alpha \ln 2 \frac{k_{B} T}{c} \sin ^{2} \theta_{i}, \\
\Pi\left(\omega, \theta_{i}\right) & \approx 8 \alpha \ln 2 \frac{k_{B} T \omega^{2}}{c^{3}} \sin ^{2} \theta_{i}
\end{aligned}
$$

and

$$
\begin{aligned}
\Pi_{00}^{*}\left(\omega, \theta_{i}\right) & \approx 8 i \alpha \ln 2 \frac{\omega_{T}}{\omega} \cos \theta_{i}, \\
\Pi^{*}\left(\omega, \theta_{i}\right) & \approx 8 i \alpha \ln 2 \frac{\omega_{T}}{\omega} .
\end{aligned}
$$

Note that in the asymptotic region (18), the pure imaginary polarization tensor at $T=0$ and the imaginary part of the thermal correction to it cancel each other out, resulting in real expressions (19). In Eqs. (21) and (22), the terms $2 \pi \alpha$ should be deleted in both numerators and denominators. The multiple $v_{F}^{2}$ in the last term of Eq. (8) should be $\tilde{v}_{F}^{2}$.

Furthermore, due to typos in the optical data for the imaginary parts of the complex index of refraction of $\mathrm{SiO}_{2}$ in the frequency region $\omega \sim 100 \mathrm{meV}$, the structure of absorption peaks in Fig. 3 is shown incorrectly. The corrected Fig. 3 is shown here.

The authors are greatly indebted to M. Antezza for attracting their attention to an incorrect structure of peaks in Fig. 3.

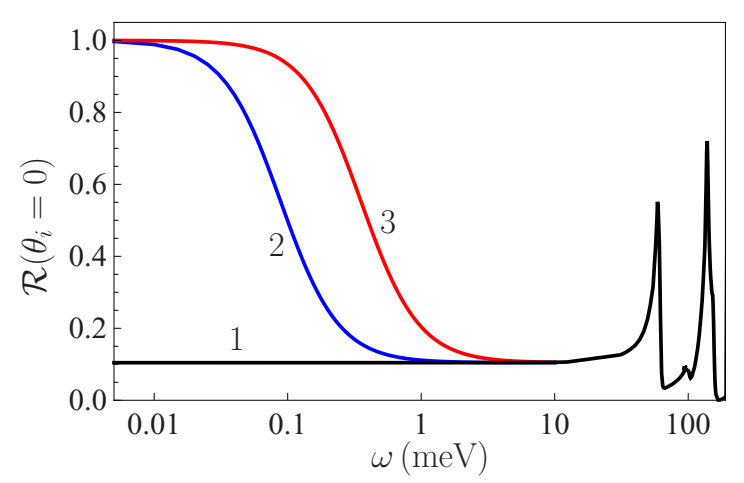

FIG. 3. The reflectivities of the graphene-coated and uncoated silica plates (lines 2, 3, and 1, respectively) at the normal incidence are shown as functions of frequency at low frequencies. Lines 2 and 3 are plotted for the graphene-coated silica at $T=75$ and $300 \mathrm{~K}$, respectively. 